\title{
Body Weight Character Result in Standard
} Format

National Cancer Institute

\section{Source}

National Cancer Institute. Body Weight Character Result in Standard Format. NCI

Thesaurus. Code C119791.

The standard character or string for representation and reporting of body weight data. 